\title{
Clinical Applications of Raman Spectroscopy in Inflammatory Bowel Diseases. A Review
}

\author{
Cristian Tefas, Marcel Tanțău
}

Iuliu Hatieganu University

of Medicine and Pharmacy, Department of Internal

Medicine,

Prof. Dr. Octavian Fodor

Regional Institute of

Gastroenterology and

Hepatology, Cluj-Napoca

Cluj-Napoca, Romania

\author{
Address for correspondence: \\ Cristian Tefas \\ Iuliu Hatieganu University \\ of Medicine and Pharmacy, \\ Department of Internal \\ Medicine, Prof. Dr. Octavian \\ Fodor Regional Institute \\ of Gastroenterology and \\ Hepatology, Cluj-Napoca, \\ Romania \\ tefascristian@gmail.com
}

Received: 22.05 .2018 Accepted: 10.10.2018

\begin{abstract}
Background \& Aims: Inflammatory bowel diseases are still difficult to diagnose and differentiate in some cases despite the serological, imaging, endoscopic and histopathological armamentarium. Raman spectroscopy is a technique that could help with these shortcomings. The aim of this paper is to present the accuracy of Raman spectroscopy in the diagnosis and monitoring of patients with inflammatory bowel diseases.

Methods: We identified the published manuscripts and abstracts up to the 31st of December 2017 by a systematic search of Medline, Embase, Cochrane and other trial registries.

Results: Eight publications were found, showing sensitivities and specificities of Raman spectroscopy in diagnosing and differentiating inflammatory bowel diseases ranging from 82 to $99 \%$ and 57 to $99 \%$, respectively, and accuracies of up to $95 \%$.

Conclusion: The technique has so far proven its potential in the positive and differential diagnosis of Crohn's disease or ulcerative colitis, allowing for very rapid results with high sensitivity and specificity.
\end{abstract}

Key words: Endoscopy - Raman spectroscopy - ulcerative colitis - Crohn's disease - inflammatory bowel disease.

Abbreviations: CD: Crohn's disease; IBD: inflammatory bowel diseases; UC: ulcerative colitis.

\section{INTRODUCTION}

Ulcerative colitis (UC) and Crohn's disease (CD) are the main well-characterized forms of inflammatory bowel disease (IBD). Their diagnosis is based on the combination of clinical, endoscopic, histopathological, serological and radiological features, but the precise distinction between UC and CD remains a challenge in 5-15\% of cases despite the technical advances in recent years At the same time, diagnosis may be difficult due to clinical overlapping between these two conditions, incomplete IBD expression, or as a result of a reduced histological response of the intestinal mucosa, so that in $5-15 \%$ of the patients the initial diagnosis is changed over time from UC to CD or vice versa Moreover, it has been reported that serological markers, such as anti-Saccharomyces cerevisiae and p-ANCA antibodies, introduced in the positive and differential diagnosis of IBD, have a high specificity but a relatively low sensitivity The differential diagnosis between UC and $\mathrm{CD}$ is mandatory due to distinct approaches in surgical treatment, differences in terms of prognosis, recurrence rate and the risk of development of neoplasia. For these reasons, it is necessary to seek new approaches in the diagnosis of IBD.

Raman spectroscopy is a technique used to quantify vibrations in a system and is based on the non-elastic diffusion of photons. It was originally developed in chemistry and used in the characterization of various molecules and their relationship, and in the physics of the solid, to describe the various characteristics of different materials.

\section{PRINCIPLES OF RAMAN SPECTROSCOPY}

The basic principle behind Raman spectroscopy relates to changing the frequency of photons after the interaction of light with an obstacle, which may be liquid, solid or gaseous in nature. After encountering the material of interest, the light is dispersed, this being determined by the characteristic absorption of the sample. If the frequency of the radiation does 
not change, a molecular equilibrium occurs, the dispersion being called Rayleigh. When the dispersion causes a photon frequency shift, the molecular vibrations undergoing changes due to the polarization of the molecules, the dispersion is called Raman, this being quantifiable in $\mathrm{cm}^{-1}$. The most intense dispersion occurs at frequency levels that are lower than the incidence of incident radiation

The importance of this technique lies in the fact that vibrational frequencies are specific to molecular chemical bonds and their symmetry. The region of interest for organic molecules is between 500 and $1500 \mathrm{~cm}^{-1}$ so that, using specific tables, reliable conclusions can be drawn on the composition of the measured sample, and the protein-lipid composition of the biological tissues can be characterized. For example, phenylalanine ring breathing corresponds to $1003 \mathrm{~cm}^{-1}, 1305$ $\mathrm{cm}^{-1}$ to $\mathrm{CH} 2$-twisting and $1660 \mathrm{~cm}^{-1}$ to amide I [5].

To obtain and quantify the Raman dispersion, the sample is radiated with monochromatic light, the most commonly used sources being lasers at various wavelengths in visible, ultraviolet, or near infrared spectra (Table I). The dispersing element is in most cases a diffraction network or an interferometer. Thus, a typical Raman system consists of a laser source, a light collection system, a wavelength selector, and a detector. The information obtained is given in the form of wavelength intensity spectra (Fig. 1).

Table I. Types of lasers used in Raman spectroscopy and their advantages

\begin{tabular}{lll}
\hline Type & Wavelength & Advantages \\
\hline $\begin{array}{l}\text { Ultra- } \\
\text { violet }\end{array}$ & $\begin{array}{l}244 \mathrm{~nm}, 257 \mathrm{~nm}, \\
325 \mathrm{~nm}, 364 \mathrm{~nm}\end{array}$ & $\begin{array}{l}\text { Ultra-violet lasers are best used } \\
\text { for detection of proteins, DNA, } \\
\text { RNA and for fluorescence } \\
\text { suppression. }\end{array}$ \\
\hline Visible & $457 \mathrm{~nm}, 473 \mathrm{~nm}$, & $\begin{array}{l}\text { Blue or green lasers are good for } \\
\text { inorganic materials and surface } \\
\text { enhanced Raman scattering. }\end{array}$ \\
& $\begin{array}{l}488 \mathrm{~nm}, 514 \mathrm{~nm}, \\
532 \mathrm{~nm}, 633 \mathrm{~nm},\end{array}$ & $\begin{array}{l}\text { Near infra-red lasers }(660-830 \\
\text { nm) are good for fluorescence } \\
\text { suppression. }\end{array}$ \\
\hline $\begin{array}{l}\text { Near infra- } \\
\text { red }\end{array}$ & $785 \mathrm{~nm}, 830 \mathrm{~nm}$, &
\end{tabular}

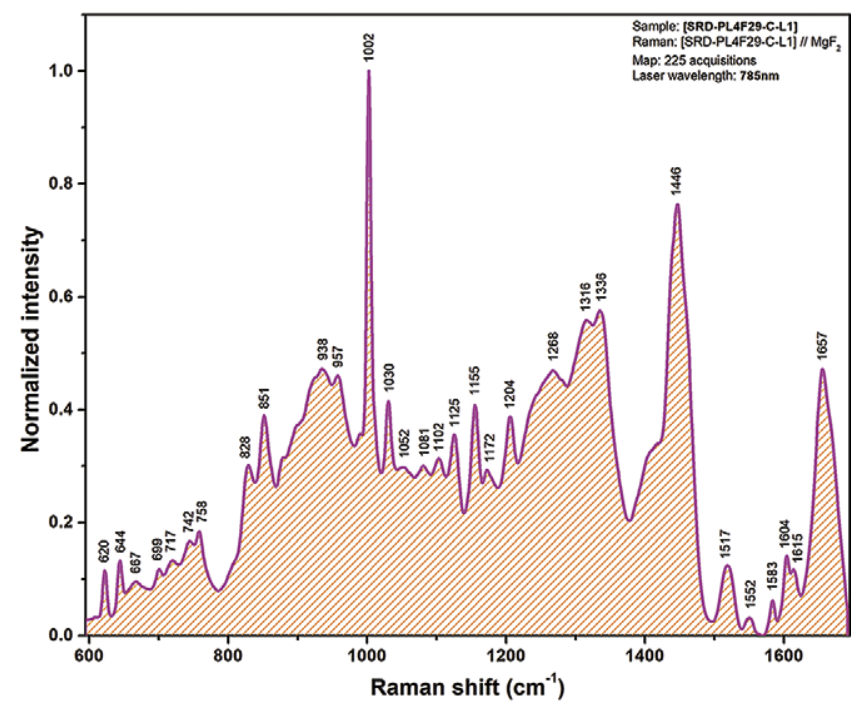

Fig, 1. A typical Raman spectra.
Compared to other analytical techniques, Raman spectroscopy offers several major advantages. It is a light scattering technique, so it does not require prior preparation of the samples, but only their placement in the irradiation area. This has a very important advantage over histological examinations requiring time for sample processing, sometimes with special and immunohistochemical stains, so that reaching a conclusive result might take a few weeks. Also, it is not necessary to dissolve the solids or otherwise alter the chemical or physical structure of the samples. The tissue samples can be analyzed in various containers, an important element when it comes to infectious materials. At the same time, since the incident light beam can be concentrated on very small regions of the order of 10 microns, the required amount of material is very small.

The major disadvantages of Raman spectroscopy are related to the quality of the laser and its wavelength. Thus, since the Raman dispersion represents $10^{-4}$ of all the dispersed light, it can sometimes be very difficult to quantify it. An inappropriate increase of laser intensity or acquisition time can lead to the destruction of the sample by burning, an undesirable event especially when working with limited quantities of the material. The use of shorter wavelengths may increase the accuracy of determinations, but sometimes cause fluorescence that masks Raman spectra.

Another thing worth mentioning is that, although the time of acquisition of a spectrum is extremely low, in the order of seconds, the total acquisition time varies greatly depending on the type of material being investigated. Thus, while within a homogeneous inorganic suspension the scanning time may remain low, because wherever the determination is made the results are similar, there is a great variation in the case of tissue samples. In this case, due to the multiple cellular and subcellular elements to be quantified separately, which have significant chemical differences, the total acquisition time may take longer and may be influenced by the size of the sample and the number of acquisitions per sample.

A major drawback of Raman spectroscopy is also the cost of the necessary equipment, which can be very high.

Raman microspectroscopy, an assembly made up of an optical microscope to which the laser system and a spectrometer are connected, has been shown to be promising for cellular and tissue diagnosis in recent years. Raman tissue spectra may be correlated with homologous histopathological examinations because the Raman tissue signature reflects its biochemical composition. The wavelengths of the lasers used in Raman microspectroscopy are 785 or $1064 \mathrm{~nm}$, which reduces the risk of sample burning at the cost of prolonged acquisition times, thus Raman dispersion is decreased towards the infrared spectra.

\section{METHOD}

We searched Medline, Embase and the Cochrane library from 1950 to 2017. The search terms used included: "Raman", "spectroscopy", "inflammatory bowel disease", "colitis" and "Crohn's disease". No language restrictions were used. No duplicates were found. We included studies using Raman spectroscopy as a diagnostic means in either UC, CD or both. 


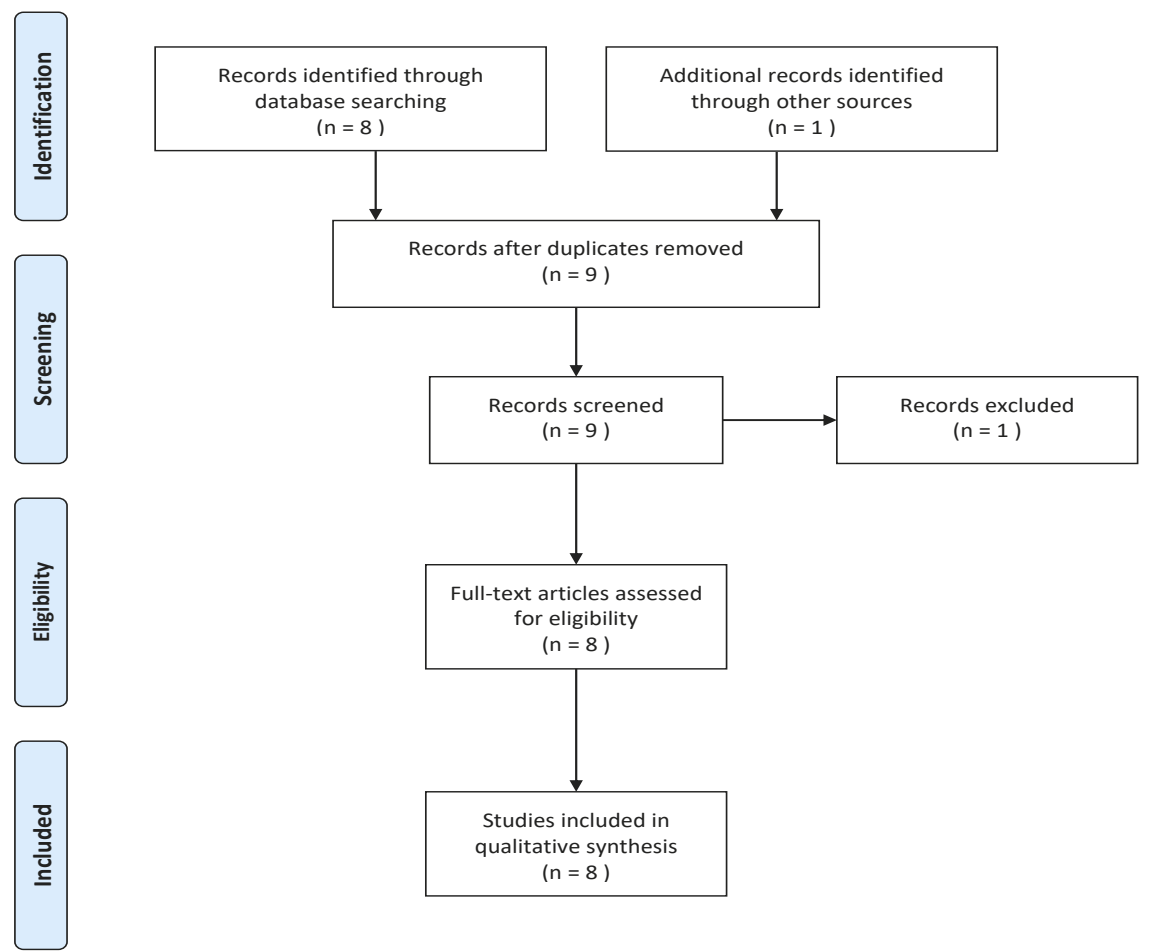

Fig. 2. Flowchart of the studies identified.

We included both animal and human studies. One study was excluded, as it did not use Raman spectroscopy as a diagnostic tool in IBD (Fig. 2).

\section{RESULTS}

Eight publications up to 2017 were found using our search criteria. All studies were performed on humans. While 5 studies were performed in vitro, using tissue samples from endoscopic biopsies or after colectomy, 3 were performed in vivo, using endoscopic-coupled Raman spectroscopy. These studies showed sensitivities and specificities of Raman spectroscopy in diagnosing and differentiating inflammatory bowel diseases ranging from 82 to $99 \%$ and 57 to $99 \%$, respectively, and accuracies of up to $95 \%$ (Table II).

\section{DISCUSSION}

The potential for Raman microspectroscopy in diagnosing multiple pathologies, including the gastroenterological ones,

Table II. Published studies on Raman spectroscopy applied in inflammatory bowel diseases

\begin{tabular}{|c|c|c|c|}
\hline Reference & Subjects & Outcomes & Performance indicators \\
\hline Bi et al., 2011 [9] & 12 pts with UC, 9 pts with CD & $\begin{array}{l}\text { Significant differences in nucleic } \\
\text { acid, phenylalanine and lipid } \\
\text { spectra }\end{array}$ & N/A \\
\hline $\begin{array}{l}\text { Bielecki et al., } 2012 \\
{[10]}\end{array}$ & $\begin{array}{l}13 \text { pts with UC, } 14 \text { pts with CD, } 11 \\
\text { healthy controls }\end{array}$ & $\begin{array}{l}\text { Accurate separation between } \\
\text { patients with IBD and healthy } \\
\text { controls }\end{array}$ & $\begin{array}{l}\text { Sensitivity } 99.07 \% \\
\text { Specificity } 98.81 \%\end{array}$ \\
\hline $\begin{array}{l}\text { Veenstra et al., } 2014 \\
{[8]}\end{array}$ & 4 pts with UC, patients without UC & $\begin{array}{l}\text { Accurate separation between } \\
\text { patients with UC and controls }\end{array}$ & $\begin{array}{ll}\text { Sensitivity } & 82 \% \\
\text { Specificity } & 89 \%\end{array}$ \\
\hline $\begin{array}{l}\text { Pence et al., } 2014 \\
{[12]}\end{array}$ & $\begin{array}{l}15 \text { pts with UC, } 26 \text { patients with CD, } 10 \\
\text { healthy controls }\end{array}$ & $\begin{array}{l}\text { Accurate real-time endoscopic } \\
\text { assessment of IBD }\end{array}$ & Accuracy $79.7 \%$ \\
\hline $\begin{array}{l}\text { Wood et al., } 2014 \\
{[14]}\end{array}$ & 18 pts with UC, 30 healthy controls & $\begin{array}{l}\text { Accurate separation of different } \\
\text { colorectal pathologies }\end{array}$ & Accuracy $95.1 \%$ \\
\hline $\begin{array}{l}\text { Chernavskaia et al., } \\
2016[11]\end{array}$ & $\begin{array}{l}7 \text { pts with UC, } 6 \text { pts with CD, } 7 \text { pts with } \\
\text { infectious colitis }\end{array}$ & $\begin{array}{l}\text { Multimodal images are able to } \\
\text { display major indicators of an } \\
\text { inflammation }\end{array}$ & N/A \\
\hline $\begin{array}{l}\text { Pence et al., } 2017 \\
{[13]}\end{array}$ & $\begin{array}{l}8 \text { pts with UC, } 15 \text { pts with } \mathrm{CD}, 8 \\
\text { healthy controls }\end{array}$ & $\begin{array}{l}\text { Accurate real-time endoscopic } \\
\text { assessment of IBD }\end{array}$ & $\begin{array}{l}\text { Sensitivity } 86 \% \\
\text { Specificity } 57 \%\end{array}$ \\
\hline $\begin{array}{l}\text { Ding et al., } 2017 \\
{[16]}\end{array}$ & 18 pts with UC, 31 healthy controls & $\begin{array}{l}\text { Accurate real-time endoscopic } \\
\text { separation between UC and } \\
\text { controls }\end{array}$ & Sensitivity $83.5 \%$ \\
\hline
\end{tabular}

pts: patients; UC: ulcerative colitis; CD: Crohns disease; N/A: not available. 
has been recently demonstrated [6, 7]. However, there is little data on the use of Raman spectroscopy to detect the molecular changes that occur in epithelial cells during inflammatory or tumoral conditions.

Inflammatory bowel diseases have been only recently studied by means of Raman spectroscopy, with the idea that this technique could reduce the number of uncertain or inaccurate diagnoses. At the same time, the possibility of developing very small optical fiber probes that can be used during endoscopic examinations and the reduced time required to obtain in vivo results could lead to a revolution in the positive and differential diagnosis and the monitoring of IBD. Thus, some authors reported promising results in diagnosing these conditions using endoscopic probes.

In one of the first studies published on the topic, using colorectal biopsy specimens from 8 patients who underwent surgery, Veenstra et al. [8] managed to differentiate subjects with UC from healthy subjects with a sensitivity of $82 \%$ and a specificity of $89 \%$, with significant differences in the spectral regions associated with nucleic acids $\left(1,044 \mathrm{~cm}^{-1}\right)$, lipids $(1,102$ and $\left.1,656-1,658 \mathrm{~cm}^{-1}\right)$ and proteins $(755,1,030-1,032,1,102$, $1,170-1,173,1,246,1,613$ and $\left.1,656-1,658 \mathrm{~cm}^{-1}\right)$, explained by the development of inflammation and increased cell turnover.

Bi et al. [9] examined colon specimens taken from 21 patients with CD and UC who underwent partial or total colectomy, in an attempt to establish differences between the two entities. Raman spectra were obtained using a laser probe emitting at $785 \mathrm{~nm}$ applied to the mucosal surface. After statistical quantification of the results, an increase in the amount of lipids (regions 1255-1314 cm $\mathrm{cm}^{-1}, 1423-1479 \mathrm{~cm}^{-1}$, $1657 \mathrm{~cm}^{-1}$ ) was demonstrated in subjects with UC versus those with $\mathrm{CD}$, more likely due to loss of lipid deposits from the submucosa in those suffering from CD. At the same time, the levels of structural proteins $\left(996-1013 \mathrm{~cm}^{-1}\right)$ and nucleic acids (1559-1576 $\mathrm{cm}^{-1}$ ) were lower in those with UC, differences that could be explained by the destruction of colon morphology and reduced cellular integrity in the examined areas. The study limits could be the lack of a control group and the use of a univariate statistical test as opposed to a multivariate one [9].

Perhaps one of the most important studies using Raman spectroscopy in the diagnosis of inflammatory bowel diseases is that of Bielecki et al. [10]. They compared spectral images obtained from 13 subjects with UC, 13 subjects with CD and 11 healthy control subjects and, using the appropriate morphological images from the same subjects in haematoxylineosin staining, developed a computerized system capable of the computational analysis of histological and spectral structures, differentiating epithelial elements from conjunctival or mucosal ones. Subsequently, using this mapping system, they were able to establish statistically significant differences between the groups of healthy and diseased subjects, especially in the regions corresponding to the heme groups $(743,1245$ and $1580 \mathrm{~cm}^{-1}$ ), which can be explained by hyperemia in the context of inflammation. Significant differences were also found in bands 1003, 1245, 1305, 1450 and $1660 \mathrm{~cm}^{-1}$, thus reinforcing the idea that spectral analysis of colon epithelium in IBD, even in clinical and endoscopic remission, can be separated from healthy epithelium.
Chernavskaia et al. [11], using multimodal nonlinear imaging techniques, including Raman spectroscopy, on human tissue biopsies from 7 patients with UC and 6 patients with $\mathrm{CD}$, highlighted important elements of inflammation of the colonic epithelium, such as the distortion of crypts, epithelial ruptures, basement membrane thickening, presence of lymphocytic infiltrates or mucosal scarring. The authors argued that with the development of sufficiently small probes that could be used in endoscopic equipment, these multimodal imaging techniques could be used in vivo as a surrogate for the histopathological examination, allowing for a rapid and independent diagnosis by the examiner. But, before an immediate spectroscopic diagnosis could be made, it is necessary to develop precise scoring methods

Taking things one step farther, Pence et al. [12] evaluated several spectra from patients with CD or UC by the Raman technique with optical fiber probes coupled to a laser using a wavelength of $785 \mathrm{~nm}$, and compared them to spectra obtained from healthy patients. All the spectra were obtained by the colonoscopic evaluation of the subjects, the probes being advanced on the endoscope operative channel. The subsequent morphological examination of the tissues in the areas of interest confirmed the subtype of IBD. There were statistically significant differences between healthy subjects and those with IBD at $425 \mathrm{~cm}^{-1}(\delta$ (CCC) skeletal backbone), $610 \mathrm{~cm}^{-1}$ (p (CH) wagging in proteins), $1080 \mathrm{~cm}^{-1}$ Of the lipids, $1440 \mathrm{~cm}^{-1}$ shoulder $(\delta(\mathrm{CH} 2)$ deformation of proteins and lipids), $1160 \mathrm{~cm}^{-1}$ and $1525 \mathrm{~cm}^{-1}$ ( $\beta$-carotene), and $1741 \mathrm{~cm}^{-1}(\nu(\mathrm{C}=\mathrm{O})$ in lipids). Overall, subjects with active disease had reduced spectra intensity, more likely in the context of parietal edema with attenuation of the intensity of incident laser beam, and widening spectra corresponding to protein elements most likely related to an increase in fibrin concentration at the level of the bowel wall. Thus, Raman spectroscopy had a sensitivity of $86 \%$ in differentiating subjects with IBD from healthy subjects with a fairly low specificity of $39 \%$, but which increased to $57 \%$ when considering the differentiation between subjects with IBD in clinical and endoscopic remission from those with a flare. At the same time, the spectroscopic examination succeeded in discriminating between the two subtypes of IBD with a sensitivity of $83 \%$ and a specificity of $55 \%$. Of course, the results can be largely influenced by the lack of homogeneity of the examined mucosa due to the presence of pathological products (blood, mucus, fibrin deposits) on its surface. This technique required great precision in handling the probe, and it was advisable to avoid exaggerated pressure on the examined mucosa.

In an attempt to improve the endoscopic diagnosis of IBD, Wood et al. [14] developed a Raman probe to be used with endoscopic equipment. However, the study was not conducted in vivo, but colonoscopic biopsy fragments from the large bowel were collected from various pathologies, including from 18 patients with $\mathrm{UC}$, which were subsequently subjected to Raman spectroscopy using an $830 \mathrm{~nm}$ laser system. After applying the appropriate statistical methods, healthy tissue was differentiated from diseased tissue with an accuracy of $95 \%$. 
However, in current clinical practice, macroscopic endoscopic differentiation between normal and pathological colorectal mucosa is readily accomplished. There are instances, among others, in some types of IBD, when the macroscopic appearance of the mucosa is perfectly normal, but there is an inflammatory activity at the microscopic level. This is important, because persistent microscopic inflammation, both acute and chronic, in patients with UC has been associated with increased relapse rate, hospitalization, colectomy and the risk of colorectal neoplasia. Depicting the microscopic inflammatory status in a macroscopically normal mucosa could lead to treatment escalation in order to reduce the risks mentioned above. Ding et al. [16] tried to achieve this goal in 18 patients with UC, using endoscopic-coupled Raman spectroscopy. They demonstrated substantial decreases in the content of total lipids and phosphatidylcholines in the inflamed colon tissue as compared to controls, as well as to those with quiescent disease, with a sensitivity of $83.5 \%$ and $97.1 \%$, respectively.

Endoscopic Raman spectroscopy could therefore be routinely added to the standard gastrointestinal endoscopy. As each Raman spectrum acquisition can be completed within 0.5 to 3 seconds, examining the whole colon adds just less than 5 minutes to the procedure, with no need for more invasive procedures, such as biopsy sampling. This is important as it greatly reduces the time it takes to provide a proper diagnosis. In addition, developing a computational system that can independently analyze samples in vivo and differentiate between healthy and diseased tissue might make sample interpretation by a pathologist or gastroenterologist unnecessary, thus reducing costs. However, with the technology presently available, the endoscopist could perform the interpretation, as long as he or she undergoes training in Raman spectra interpretation.

Another possible indication in using Raman spectroscopy is the endoscopic surveillance of patients with colonic IBD, as we know that colorectal cancer has a higher incidence in these patients compared to healthy subjects. In a study including 65 patients with Barrett's esophagus, Raman spectroscopy identified lesions containing high-grade dysplasia or early cancer with an overall diagnostic accuracy of $89 \%$ [18]. As such, the same method could be applied to patients with IBD. Highlighting areas with high-grade dysplasia in the colon before they can be visualized with the current techniques could completely change the management of these patients, and drastically reduce the need for high-risk endoscopic or surgical interventions.

\section{CONCLUSION}

We are at an important crossroads in the development of positive and differential diagnosis of IBD. Raman spectroscopy demonstrates important advantages in relation to the speed and accuracy of the results, with sensitivities and specificities ranging from 82 to $99 \%$ and 57 to $99 \%$, respectively. In the future, histopathological examination might become unnecessary, as endoscopic tissue spectral analysis yields immediate results. In addition, Raman spectroscopy can be performed on fluids, so that in the future we might not even require endoscopic exploration in patients with IBD, establishing the diagnosis and performing the monitoring by using simple blood tests.

Conflict of interests: No conflict to declare.

Authors' contributions: C.T. drafted and wrote the article. M.T. revised the article.

\section{REFERENCES}

1. Guindi M, Riddell RH. Indeterminate colitis. J Clin Pathol 2004;57:1233-1244. doi:10.1136/jcp.2003.015214

2. Henriksen M, Jahnsen J, Lygren I, et al. Change of diagnosis during the first five years after onset of inflammatory bowel disease: results of a prospective follow-up study (the IBSEN Study). Scand J Gastroenterol 2006;41:1037-1043. doi:10.1080/00365520600554527

3. Peeters M, Joossens S, Vermeire S, Vlietinck R, Bossuyt X, Rutgeerts P. Diagnostic value of anti-Saccharomyces cerevisiae and antineutrophil cytoplasmic autoantibodies in inflammatory bowel disease. Am J Gastroenterol 2001;96:730-734. doi:10.1111/j.1572-0241.2001.03613.x

4. UK Essays. Raman Spectroscopy [Internet]. November 2013. [Accessed 10 October 2017]; Available from: https://www.ukessays.com/essays/ sciences/raman-spectroscopy.php?cref $=1$

5. De Gelder J, De Gussem K, Vandenabeele P, Moens L. Reference database of Raman spectra of biological molecules. J Raman Spectrosc 2007;38:1133-1147. doi:10.1002/jrs.1734

6. Kong K, Kendall C, Stone N, Notingher I. Raman spectroscopy for medical diagnostics--From in-vitro biofluid assays to in-vivo cancer detection. Adv Drug Deliv Rev 2015;89:121-134. doi: 10.1016/j. addr.2015.03.009

7. Beleites C, Bonifacio A, Codrich D, Krafft C, Sergo V. Raman spectroscopy and imaging: promising optical diagnostic tools in pediatrics. Curr Med Chem 2013;20:2176-2187. doi:10.2174/0929867311320170003

8. Veenstra MA, Palyvoda O, Alahwal H, et al. Raman Spectroscopy in the Diagnosis of Ulcerative Colitis. Eur J Pediatr Surg 2014;25:56-59. doi:10.1055/s-0034-1387951

9. Bi X, Walsh A, Mahadevan-Jansen A, Herline A. Development of spectral markers for the discrimination of ulcerative colitis and Crohn's disease using Raman spectroscopy. Dis Colon Rectum 2011;54:48-53. doi:10.1007/DCR.0b013e3181fff68d

10. Bielecki C, Bocklitz TW, Schmitt M, et al. Classification of inflammatory bowel diseases by means of Raman spectroscopic imaging of epithelium cells. J Biomed Opt 2012;17:076030. doi:10.1117/1.JBO.17.7.076030

11. Chernavskaia O, Heuke S, Vieth M, et al. Beyond endoscopic assessment in inflammatory bowel disease: real-time histology of disease activity by non-linear multimodal imaging. Sci Rep 2016;6:29239. doi:10.1038/ srep29239

12. Pence IJ, Beaulieu DB, Horst SN, et al. Endoscopy-coupled Raman spectroscopy for in vivo discrimination of inflammatory bowel disease. Proc. SPIE 8939, Biomedical Vibrational Spectroscopy VI: Advances in Research and Industry, 89390R (4 March 2014). doi:10.1117/12.2042795

13. Pence IJ, Beaulieu DB, Horst SN, et al. Clinical characterization of in vivo inflammatory bowel disease with Raman spectroscopy. Biomed Opt Express 2017;8:524-535. doi:10.1364/BOE.8.000524

14. Wood JJ, Kendall C, Hutchings J, et al. Evaluation of a confocal Raman probe for pathological diagnosis during colonoscopy. Colorectal Dis 2014;16:732-738. doi:10.1111/codi.12664

15. Bryant RV, Winer S, Travis SP, Riddell RH. Systematic review: histological remission in inflammatory bowel disease. Is 'complete' 
remission the new treatment paradigm? An IOIBD initiative. J Crohns Colitis 2014;8:1582-1597. doi:10.1016/j.crohns.2014.08.011

16. Ding H, Dupont AW, Singhal S, et al. In vivo analysis of mucosal lipids reveals histological disease activity in ulcerative colitis using endoscopecoupled Raman spectroscopy. Biomed Opt Express 2017;8:3426-3439. doi:10.1364/BOE.8.003426
17. Kim ER, Chang DK. Colorectal cancer in inflammatory bowel disease: The risk, pathogenesis, prevention and diagnosis. World J Gastroenterol 2014;20:9872-9881. doi:10.3748/wjg.v20.i29.9872

18. Wong Kee Song LM, Molckovsky A, Wang KK, et al. Diagnostic potential of Raman spectroscopy in Barrett's esophagus. Proc SPIE 2005;5692:140-146. doi:10.1117/12.584986 